\title{
An empirical analysis of dialect and regional economic growth based on OLS regression model
}

\author{
Wang Simin*1,a,Li Jiayuan ${ }^{2, b}$,Guo Wenjie ${ }^{3, c}$,Yang Huilin ${ }^{4, d}$, Fei Yang ${ }^{5, e}$ \\ ${ }^{1}$ Sichuan Agricultural University Chengdu, China \\ ${ }^{2}$ Sichuan Agricultural University Chengdu, China \\ ${ }^{3}$ Sichuan Agricultural University Chengdu, China \\ ${ }^{4}$ Sichuan Agricultural University Chengdu, China \\ ${ }^{5}$ Sichuan Agricultural University Chengdu, China
}

\begin{abstract}
There is a relatively new economic growth theory that culture is a deeper force to explain economic growth. This paper will establish an economic growth model based on Solow model, and discuss the economic growth of multi-ethnic areas from the perspective of language economics. On the basis of literature research, this paper first puts forward the conjecture that dialect diversity has a negative impact on the economic income level of multi-ethnic areas. In order to verify this conjecture, this paper selects Xiaojin County, Danba County, Puge county and Butuo County of Sichuan Province as the investigation objects. After screening more than 200 effective data, the least square linear regression model is used to carry out empirical analysis. Through White Heteroscedasticity Test and WLS Heteroscedasticity Test, the hypothesis that dialect diversity has a negative impact on economic income level in multi-ethnic areas is verified.
\end{abstract}

\section{Perface}

Sichuan Province is a multi-ethnic province in Southwest China, covering a total area of 486000 square kilometers, including three autonomous prefectures and four autonomous counties, with 183 county-level divisions, including 51 Minority Counties, covering more than $60 \%$ of the total area of the province. There are 56 ethnic minorities and 12 ethnic minorities in the province, of which the number of ethnic minorities accounts for $6.47 \%$ of the total population of the province. The multi-ethnic areas are often distributed in the mountainous, hilly and plateau terrain complex belt, with poor traffic convenience and backward economic level. Diversified ethnic distribution leads to diversified ethnic languages. According to the classification of language genealogy, except for the languages used by Manchu and Mongol, the national languages distributed in various regions of Sichuan province belong to the Sino Tibetan language family, with a total of 21 kinds. However, in March of 2018, the Provincial Department of education, the poverty alleviation Bureau and the language Working Committee issued a document on "the implementation plan of Sichuan Province to promote the national common language and characters to help poverty alleviation", which focused on more than 80 poverty-stricken counties, filing and Lika poverty-stricken villages and poor households in Sichuan Province, including Tibetan area, Liangshan Prefecture, Aba Prefecture, Ganzi Prefecture and three districts. Relevant departments actively cooperate, vigorously a*wsm7987@163.com

b1486136590@qq.com c164712699@qq.com d1602578046@qq.com e984408672@qq.com popularize the national common language, and strive to do a good job in the project of "pushing the general out of poverty". Therefore, language has become an important factor in the process of economic development. Whether the more common language can play a role as a lubricant in the economic activities such as commodity exchange and service trade has become an irrecoverable factor in the process of economic development Avoid problems. Based on the investigation of dialect diversity in multi-ethnic areas of Sichuan Province, the related literature and the first-hand statistics, this paper will study whether language diversity will affect economic growth and the degree of its impact on economic growth.

\section{Literature Review}

China is a multi-ethnic country with rich language resources. According to incomplete data, there are more than 120 kinds of national languages found among 55 ethnic minorities in China. As an important communication tool, language is the main way of communication, which plays a role in preserving and transmitting human civilization, and is one of the important characteristics of a nation. In addition, the German scholar Dustmann ${ }^{[1]}$ has shown that there is an important relationship between German proficiency and the working class's remuneration and working level; similar studies conducted by bloom and Grenier ${ }^{[2]}$ in the United States, Vaillancourt ${ }^{[3]}$ in Canada, and Beenstock, Chiswick and repetto ${ }^{[4]}$ in Israel have also confirmed that 
there is a positive correlation between language proficiency and migrant income Correlation. Zhang Weiguo $^{[5]}$, a domestic scholar, also pointed out that language is a kind of human capital. He believes that language plays an important role in determining employment in the labor market and people's labor income. Investment in language skills can bring short-term and long-term benefits, especially in multi-ethnic areas. Therefore, there is an inseparable relationship between language and economy, and the optimization of language is of great significance to the improvement of economic level. However, in the face of diversified languages, scholar Mo Zaishu ${ }^{[6]}$ pointed out that different languages have different contribution values to economic development, and different languages have different economic values. Lei Xiaolan ${ }^{[7]}$ believes that common and strong language can reduce the cost of unnecessary information collection, analysis and exchange, enhance the trust between the two sides of communication, reduce the transaction risk, bring direct benefits to the user group, and help expand the business types of indirect income. $\mathrm{Xu}$ Xianxiang ${ }^{[8]}$ used the data of cities above the national level to conduct research and analysis, and concluded that the higher the degree of dialect diversification, the lower the level of economic development. However, in multi-ethnic areas, similar research is still relatively scarce. Whether the diversity of language is hindering economic growth, especially in multi-ethnic areas, what kind of relationship will be shown between language diversity and economic growth, and the degree of impact on economic level is a question. And the solution of these problems will help to better balance the relationship between economic development and the protection of ethnic cultural diversity, which is of great significance to the economic development and planning of ethnic areas and the formulation of language policies.

\section{Dialect Diversity And Economics Growth}

According to the statistics of the dictionary of Chinese dialects, there are 17 dialects and 105 sub dialects in China. From the perspective of languages used by various ethnic groups in Sichuan Province, Liu Huiqiang (1984) pointed out that except for Hui and Manchu, other ethnic minorities have their own languages, and some even use more than one language. From the perspective of economic data, although the "three prefectures" of Sichuan Province account for $60.3 \%$ of the total area of Sichuan Province, the total economic volume only reaches $5.3 \%$ of the whole province. In recent years, the government has actively organized the work of "language poverty alleviation". According to the survey data, less than $30 \%$ of the families in ethnic areas use Putonghua at home.
On the basis of the above, this paper puts forward the hypothesis: the more diverse the dialect, the lower the economic level.

In macroeconomic research, economists use production function to analyze the relationship between various factors and explain economic growth. There is a relatively new economic growth theory that culture is a deeper force to explain economic growth. Therefore, in order to further analyze the impact of language diversity on economic growth, this paper will build a model (1).

$$
\ln \mathrm{y}_{\mathrm{i}}=\alpha_{1}+\alpha_{2} \operatorname{Lan}+X_{i} \psi_{i}+\varepsilon_{i}
$$

In this paper, LAN is used to express the number of sub dialects that the surveyed families are exposed to in their places of life. The minimum value of LAN is 1 . The more diverse the language, the greater the value of LAN. $y$ represents the economic income of the family. ${ }^{\ln } y_{i}$ is the logarithm of the household income of the observation sample; LAN is the dialect diversity index of the region; $X_{\mathrm{i}}$ is a series of factors that don't change over time, including Dis-county, Edu, Type. Dis-county is the distance between the sample family and the county seat, calculated by the highway distance, and its unit is km; Edu represents the education level of the main labor force in the sample family, the Edu value is between 1 and 5, the higher the Edu value is, the higher the education level is; Type is a virtual variable, indicating whether the type of household is urban or rural. $\varepsilon$ is the disturbance term. ${ }^{\alpha_{2}}$ is the coefficient of LAN value in formula (1), which is used to measure the influence direction and degree of dialect diversity on economic growth, If the theoretical hypothesis holds, then $\alpha_{2}<0$.

\subsection{Empirical Analysis}

This paper selects Xiaojin, Danba, Kangding, Luding, Puge and Butuo counties as the survey sites of multiethnic areas by consulting relevant documents and materials. In July of 19 years, I had field interview and questionnaire survey to obtain the first-hand data and data of local dialect use, family language use habits and family economic status. The number of effective observations in this sampling survey is 173 . Of the 173 families surveyed, 73 were in multi-ethnic areas or "dialect complex counties", accounting for $42.2 \%$ of the total sample. The remaining 100 families came from non multi-ethnic areas or "dialect complex counties". Table 1 is a simple descriptive statistics of dialect diversity. As shown in Table 1, the average value of LAN in 173 families is 1.85 , the minimum value is 1 , and the maximum value is 4 . That is to say, the number of sub dialects that families touch at the same time in daily life is up to 4 .

Table1. Simple description statistics of dialect diversity

\begin{tabular}{|c|c|c|c|c|c|c|c|c|}
\hline Project & Mean & Minimum & $25 \%$ quantile & $50 \%$ quantile & $75 \%$ quantile & Maximum & $\sigma$ & Quantity \\
\hline Lan & 1.85 & 1 & 1 & 2 & 2 & 4 & 0.9025 & 173 \\
\hline
\end{tabular}


Table 2 shows the results obtained after regression with formula (1). The regression coefficient of LAN is always less than zero and remains significant, so the

Table2. Influence of dialect diver

\begin{tabular}{|c|c|c|c|c|}
\hline \multirow{2}{*}{ Variable } & $(1)$ & $(2)$ & (3) & $(4)$ \\
\hline & \multicolumn{4}{|c|}{$\ln y$} \\
\hline Lan & $\begin{array}{c}-0.1657938^{* * *} \\
(0.0572411)\end{array}$ & $\begin{array}{c}-0.1547854^{* * * *} \\
(0.0546876)\end{array}$ & $\begin{array}{c}-0.1449492 * * \\
(0.056157)\end{array}$ & $\begin{array}{r}-0.1276836^{* *} \\
(0.0573852)\end{array}$ \\
\hline Dis-county & - & $\begin{array}{l}- \\
-\end{array}$ & - & $\begin{array}{l}-0.0085421 * \\
(0.0050899)\end{array}$ \\
\hline Constant & $\begin{array}{l}11.43591 * * * \\
(0.0948188)\end{array}$ & $\begin{array}{l}11.28165^{* * * *} \\
(0.1042818)\end{array}$ & $\begin{array}{c}11.09051^{* * *} \\
(0.155457)\end{array}$ & $\begin{array}{l}11.20968^{* * *} \\
(0.1617883)\end{array}$ \\
\hline Type & $\begin{array}{l}- \\
-\end{array}$ & $\begin{array}{c}0.2790803 * * * \\
(0.0825903)\end{array}$ & $\begin{array}{c}0.2289003 * * \\
(0.08908)\end{array}$ & $\begin{array}{c}0.1487397 \\
(0.0986887)\end{array}$ \\
\hline Edu & - & - & $\begin{array}{c}0.065171 * * \\
(0.031595)\end{array}$ & $\begin{array}{l}0.0531841^{*} \\
(0.0316479)\end{array}$ \\
\hline$R^{2}$ & 0.0669 & 0.1251 & 0.1421 & 0.1612 \\
\hline Observations & 173 & 173 & 173 & 173 \\
\hline
\end{tabular}

Note: the regression results in the above table are estimated by OLS; in brackets are the robust standard errors corrected by white heteroscedasticity; * $* *$ indicates that the $\mathrm{p}$ value is less than $0.01, * *$ indicates that the $\mathrm{p}$ value is less than $0.05, *$ indicates that the $\mathrm{p}$ value is less than 0.1 ; $\mathrm{R} 2$ is the unadjusted judgment coefficient.

The first column of Table 1 reports the results of oneway regression of LAN, which only introduces the diversity of dialects. At this time, the regression coefficient of LAN is -0.166 , which can pass the test of $1 \%$ significance level. This shows that for every unit of decline in dialect diversity, the economic income will increase by $16.6 \%$. Specifically, according to the descriptive statistics in Table 1 , the maximum and minimum values of LAN are 4 and 1 respectively. If the diversity of urban dialects is eliminated, the maximum economic income can increase by $49.8 \%$ when other conditions remain unchanged. Columns $2-4$ in Table 1 report the regression results after gradually introducing relevant control variables. Specifically, columns 2-4 gradually introduce the virtual variable of residents type of sample families, the distance between the education level of the main labor force in the family and the distance between the family and the county. Column 5 of Table 1 reports the regression results after the introduction of these control variables at the same time. At this time, the regression coefficient of LAN is still a negative sign, $\alpha_{2}$ $<0$. It can pass the statistical test with a significance level of $5 \%$. The absolute value is slightly smaller, from about 0.145 to 0.128 . It shows that if the diversity of dialects is hypothesis that the more diverse the dialect types in this paper, the lower the economic level is holds. eliminated and other conditions remain unchanged, the maximum economic income can be increased by about $38.4 \%$. As far as the control variables are concerned, the regression coefficient of the distance between the sample family and the county is about 0.00854 , which can pass the test with a significant level of $10 \%$. The unit is $\mathrm{km}$, that is, the closer the family is to the county, the higher the average economic income is. When other conditions remain unchanged, the average economic income will increase by $0.854 \%$ for every $100 \mathrm{~km}$ less distance. The minimum value of edu is 1 , the maximum value is $5,1-5$ represents the education level of none, primary school, junior high school, senior high school, University and above. As shown in Table 2, the regression coefficient of the education level of the main labor force in the family is about 0.0531 , which can pass the test with a significant level of $10 \%$. So if other conditions remain unchanged, the income of the main labor force in the family will increase by $5.31 \%$ for each additional level of education level.

All in all, the above regression results are consistent with the theoretical hypothesis expected in this paper. Dialect diversity has a significant negative impact on economic growth. In the above simulation scenario, if the dialect diversity is eliminated, the economic income can be increased by $49.8 \%$ at most.

Table3. Impact Of Dialect Diversity On Economic Development Level By WLS Estimation

\begin{tabular}{lcccc}
\hline \multirow{2}{*}{ variable } & \multicolumn{2}{c}{$(1)$} & \multicolumn{3}{c}{$(3)$} & $(4)$ \\
\cline { 2 - 5 } Lan & $-0.2024081^{* * *}$ & $-0.1877923^{* * *}$ & $-0.1714562 * * *$ & $-0.159322^{* * *}$ \\
\cline { 2 - 5 } & $(0.0515446)$ & $(0.0495069)$ & $(0.0497292)$ & $(0.0503952)$ \\
\hline \multirow{2}{*}{ Dis-county } & - & - & - & -0.0063949 \\
\hline \multirow{2}{*}{ Constant } & - & - & 11.17197 & $(0.00469)$ \\
\hline \multirow{2}{*}{ Type } & $(0.0827972)$ & $(0.0936148)$ & $(0.1343668)$ & $(0.1460899)$ \\
\hline \multirow{2}{*}{ Edu } & - & $0.2261867 * * *$ & $0.172452^{* *}$ & 0.123293 \\
\hline & - & $(0.0729657)$ & $(0.0770631)$ & $(0.0849028)$ \\
\hline
\end{tabular}




\begin{tabular}{lcccc}
\hline$R^{2}$ & 0.0827 & 0.1351 & 0.1555 & 0.1647 \\
\hline Observations & 173 & 173 & 173 & 173 \\
\hline
\end{tabular}

Note: $* * *$ indicates that the $\mathrm{p}$ value is less than $0.01, * *$ indicates that the $\mathrm{p}$ value is less than $0.05, *$ indicates that the $\mathrm{p}$ value is less than $0.1 ; \mathrm{R} \wedge 2$ is the unadjusted decision coefficient.

Considering the existence of heteroscedasticity, this paper makes WLS estimation for the above model again, and the results are shown in Table 3. The first column of Table 3 is the regression result when the independent variable is only LAN of dialect diversity. The second to fourth columns are the regression results after introducing dummy variable of resident type of sample family, education level of main labor force in family and distance between family and county on the basis of cited variable LAN. It can be seen that the regression coefficient of LAN is always negative and has passed the $1 \%$ significance test.

Specifically, in the case of only independent variable LAN in the first column of Table 3 , the regression coefficient of LAN is -0.202 . One unit of dialect diversity decreases, the economic income will increase by about $20.2 \%$. In the second column of Table 3, both LAN and type can pass the test of significance level of $1 \%$. At this time, the absolute value of regression coefficient of LAN slightly decreases. However, every unit of decrease in LAN value will still bring about $18.8 \%$ positive impact on economic income. In the third column of Table 3, after introducing the education level of the main labor force in the family, LAN, type and edu can pass the significance test of $1 \%, 5 \%$ and $5 \%$ respectively, and each decrease of LAN value can bring about $17.1 \%$ positive impact on economic income. In the fourth column of Table 3, after introducing the distance between family and county, the regression coefficient of LAN value is -0.159 , which can still pass the significance level of $1 \%$. According to table 1 , if the above control variables are introduced, and the dialect diversity is eliminated and other conditions remain unchanged, the economic income can increase by about $47.7 \%$.

In a word, the value of Lan s was always negative and could pass the test. That is to say, the conclusion that dialect diversity has a significant negative impact on economic growth is still valid when considering heteroscedasticity. In the simulation of the above assumptions, if the related variables are controlled, the elimination of dialect diversity can bring about $47.7 \%$ positive impact on economic income.

\section{Conclusion}

In the simulation of the above assumptions, by constructing regression model and estimating OLS and WLS, it can be concluded that dialect diversity in multiethnic areas of Sichuan Province has a significant negative impact on the economic income level of families. At present, the research on the economic relevance of dialects in China is still relatively scarce, especially in the multilanguage areas of ethnic minorities. On the one hand, these minority areas with rich language resources are facing the demands of poverty alleviation and economic development, and on the other hand, the problem of protecting ethnic cultural diversity is prominent. Dialect, as an important carrier of Chinese national culture, its inheritance is of course important, but under the market form of general economy, the diversity of dialect to a certain extent plays a restraining role in the regional economy. Therefore, through this analysis, this paper suggests that the government should pay attention to the economic value of language, actively promote the "promotion and poverty alleviation", do bilingual education and language planning in many ethnic areas, while protecting local dialects and carrying out the inheritance of language and character heritage, and ensure the stability of economic growth.

\section{Acknowledgment}

Fund Project: Sichuan Province Innovation Training Program (No. 201910626084).

\section{References}

1. Dustmann, C.1994. Speaking fluency, writing fluency and earnings of migrants[J]. Journal of Population Economics (7):133-156.

2. Bloom, D.E.\& Grenier, G. Language, employment, and earnings in the United States: Spanish-English differentials from 1970 to 1990. International Journal of the Sociology of Language, 121(1), 1996， 45-68.

3. Vaillancourt, F.1996.Language and socioeconomic status in Quebec: Measurement, findings, determinants, and policy costs $[\mathrm{J}]$. International Journal of the Sociology of Language 121(1): 69-92.

4. Beenstock, M., Chiswick, B. R., \& Repetto, G. L. (2001). The effect of linguistic distance and country of origin on immigrant language skills: application to Israel. International Migration,39(3),33-60.

5. Zhang Weiguo. Language as human capital, public goods and institutions: a basic analytical framework of linguistic economics [J]. Economic research, 2008 (02): 144-154。

6. Mo Zaishu, Zhang Xiaoyong, Zhang Yun. 2006. business English Research Based on linguistic economics [J]. Journal of Hunan University (SOCIAL SCIENCE EDITION) (4): 102-106.

7. Lei Xiaolan. Analysis of the economic value of language $[\mathrm{J}]$. Journal of Xi'an Jiaotong University (SOCIAL SCIENCE EDITION), 2009,29 (06): 107110.

8. Xu Xianxiang, Liu Yuyun, Xiao Zekai. Dialect and economic growth [J]. Journal of economics, 2015,2 (02): 1-32.

9. Liu Huiqiang. Languages and characters of ethnic minorities in Sichuan Province [J]. JOURNAL OF SOUTHWEST UNIVERSITY FOR NATIONALITIES (PHILOSOPHY AND SOCIAL SCIENCES), 1984 (03): 35-38 + 100. 